\title{
Adaptive Morphological Filtering Using Similarities Based on Geodesic Time
}

\author{
Jacopo Grazzini and Pierre Soille \\ Spatial Data Infrastructures Unit \\ Institute for Environment and Sustainability \\ Joint Research Centre - European Commission \\ TP 262 - via E.Fermi, 2749 - 21027 Ispra (VA), Italy \\ $\{$ Jacopo.Grazzini, Pierre.Soille\}@jrc.it
}

\begin{abstract}
In this paper, we introduce a novel image-dependent filtering approach derived from concepts known in mathematical morphology. Like other adaptive methods, it assumes that the local neighbourhood of a pixel contains the essential process required for the estimation of local properties. Indeed, it performs a local weighted averaging by combining both spatial and tonal information in a single similarity measure based on the local calculation of discrete geodesic time functions. Therefore, the proposed approach does not require the definition of any initial spatial window but determines adaptively, directly from the input data, the neighbouring sample points and the associated weights. The resulting adaptive filters are consistent with the content of the image and, therefore, they are particularly designed for the purpose of denoising and smoothing of digital images.
\end{abstract}

\section{Introduction}

The dictionary of computer vision [1] states that adaptivity is the property of an algorithm to adjust its parameters to the data at hand in order to optimise performance. In this context, adaptive filtering, in which the parameters of the filter vary over different parts of the data, is a suitable approach for advanced image processing [2]. Indeed, the need for an adaptive approach to cope with inhomogeneities in images is well recognized [34]: adaptive filtering is a class of typical nonlinear smoothing techniques that has been applied to many computer vision tasks 56778 .

Strong relations have been established between a number of widely-used adaptive filters for digital image processing [9]. It has been shown that images filtered using adaptive neighbourhoods are superior to those filtered using fixed neighbourhoods, as the adaptive-neighbourhood techniques tune themselves to the contextual details in the image [4. Indeed, the use of spatially adaptive approaches implies that operators must vary over the whole image, taking into account the local image context. It assumes intrinsically that the neighbourhood of a pixel contains the essential process required for local estimation of its true intensity value. Adaptive operators can be subdivided in two main classes: the

D. Coeurjolly et al. (Eds.): DGCI 2008, LNCS 4992, pp. 519-528, 2008.

(C) Springer-Verlag Berlin Heidelberg 2008 
adaptive-weighted operators and the spatially-adaptive operators [810], where the adaptive concept results respectively from the adjustment of the weights upon the operational window and from the spatial adjustment of the window. The operator of the first class typically involves convolution between the image and a fixed-size sliding window positioned over each pixel whose coefficients are dependent on the image statistics under the sliding window. The adaptive neighbourhood of the second class surrounds the central pixel to be filtered, but its shape and the area it covers are dependent on the local characteristics of the image rather than being arbitrarily defined. Besides, some recently developed techniques propose the use of a non-local approach for filtering, where the neighbourhoods used in the estimation are not necessarly spatially connected to the central pixel 11 .

In the context of mathematical morphology (MM), the adaptive paradigm proposes solutions to the limitations of classical morphological transformations in the absence of any prior knowledge about the analysed images. By adopting an adaptive strategy, one addresses the lack of flexibility of morphological operators based on the difficult choice of a structuring element [12. Several morphological techniques have been developed to take into account the local features of the image. In [13], adaptive-neighbourhood sequential filters are proposed, where neighbourhood of increasing size are calculated for each pixel using a similarity criterion and a region-growing approach. In [10, spatially adaptive operators using operational windows, whose extent is defined around each pixel by the connected regions for a selected criterion mapping (e.g., luminance, local contrast, or local curvature), are built. In [14], similar neighbourhoods are calculated by introducing at every image pixel a distance defined between the values of the image only. Pixels reached within this adaptive neighbourhood are referred to as an amoeba; the mean or rank statistics are then computed within the amoeba to filter the image. Another approach to investigate the morphology of the image structures is to define them as connected components of pixels satisfying some properties [151617.

In this paper, we propose an image-dependent filtering approach also aiming at extending the well-defined concepts of MM in order to consider radiometric, geometrical and morphological characteristics of the images 810 18. The main idea is to associate with each pixel of the input image a weighted convolution of sample points within an adaptive neighbourhood, where the weights depend not only on the points location but also on their greylevel distance to the central pixel. For that purpose, we define some local similarity measure of the twofold spatial and tonal information based on the local estimation of pairwise discrete geodesic time functions in the local neighbourhood of the central pixel. This approach can be seen as an extension of [19], where a geodesic dilation was obtained by thresholding the generalised geodesic time function computed within the mask of greylevels. It can also be related to the concept of amoeba. Similarly, the method we introduce does not require the definition of any initial spatial window as it determines the neighbouring sample points directly. Besides, it is also able to estimate adaptively the associated weights from the input data. 
The rest of the paper is organised as follows. In the next section, we recall the fundamental notions of discrete geodesic path and geodesic time known in MM, and the way adaptive neighbourhoods can be defined. In section 3, we propose two different filtering approaches based on the estimation of local geodesic time. We also precise the filtering strategy for the case of multispectral images. The conclusion and a description of future foreseen developments are presented in section 4

\section{Geodesic Time and Adaptive Neighbourhoods}

\subsection{Discrete Geodesic Time}

Geodesic transforms are classical operators in discrete image analysis 2012122]. The geodesic distance between two pixels of a discrete connected set (typically, a binary image) is defined as the length of the shortest path(s) linking these points and remaining in the set (the so-called geodesic paths) 12]22. This idea can been generalised to greylevel images using the geodesic time on geodesic mask 19/23. The geodesic mask image is then treated as a 'height map', i.e. a surface embedded in a 3D space, with the third coordinate given by the greylevel values.

Formally, we define a discrete path $\mathcal{P}$ of length $l-1$ going from $\mathbf{p}$ to $\mathbf{q}$ as a $l$-tuple $\left(\mathbf{x}_{1}, \ldots, \mathbf{x}_{l}\right)$ of pixels such that $\mathbf{x}_{1}=\mathbf{p}, \mathbf{x}_{l}=\mathbf{q}$, and $\left(\mathbf{x}_{i-1}, \mathbf{x}_{i}\right)$ defines adjacent pixels for all $i \in[2, l]$. Introducing the greylevel geodesic mask $g$, the time $\tau_{g}(\mathcal{P})$ necessary to cover $\mathcal{P}$ represents the sum of the greylevel values of the pixels along $\mathcal{P}$ [19]. This assumes the $\operatorname{cost} c_{i}$ of travelling from a pixel $\mathbf{x}_{i}$ to an adjacent pixel $\mathbf{x}_{i+1}$ to be:

$$
c_{i}=\frac{1}{2}\left(g\left(\mathbf{x}_{i}\right)+g\left(\mathbf{x}_{i+1}\right)\right) \cdot\left|\mathbf{x}_{i}-\mathbf{x}_{i+1}\right|
$$

Here, the spatial distance $\left|\mathbf{x}_{i}-\mathbf{x}_{i+1}\right|$ refers to the elementary step in the image graph: it is chosen as either the Euclidean distance or the optimal Chamfer propagating weights in a binary $3 \times 3$ mask 24 . Intuitively, the lower the intensity value in $g$, the faster the propagation. The geodesic time $\tau_{g}(\mathbf{p}, \mathbf{q})$ separating two points $\mathbf{p}$ and $\mathbf{q}$ is then the smallest amount of time allowing to link $\mathbf{p}$ to $\mathbf{q}$ in $g$, i.e. it consists in finding the path with the lowest sum of greylevel values along all possible discrete paths linking $\mathbf{p}$ to $\mathbf{q}[19$ :

$$
\tau_{g}(\mathbf{p}, \mathbf{q})=\min \left\{\tau_{g}(\mathcal{P}) \mid \mathcal{P} \text { is a path linking } \mathbf{p} \text { to } \mathbf{q}\right\} .
$$

This concept is closely related to the notion of grey weighted distance transform defined in [25] and to the continuous framework of the minimal path approach of 26. It leads to efficient algorithms because classical shortest path algorithms can be applied such as the Dijkstra's graph search algorithm. Typically, algorithms based on priority queue data structures [12]27] enable the implementation of the local geodesic time with a computational complexity of $O(n \log n)$, where $n$ is the number of pixels in the considered spatial domain 22 27. They 

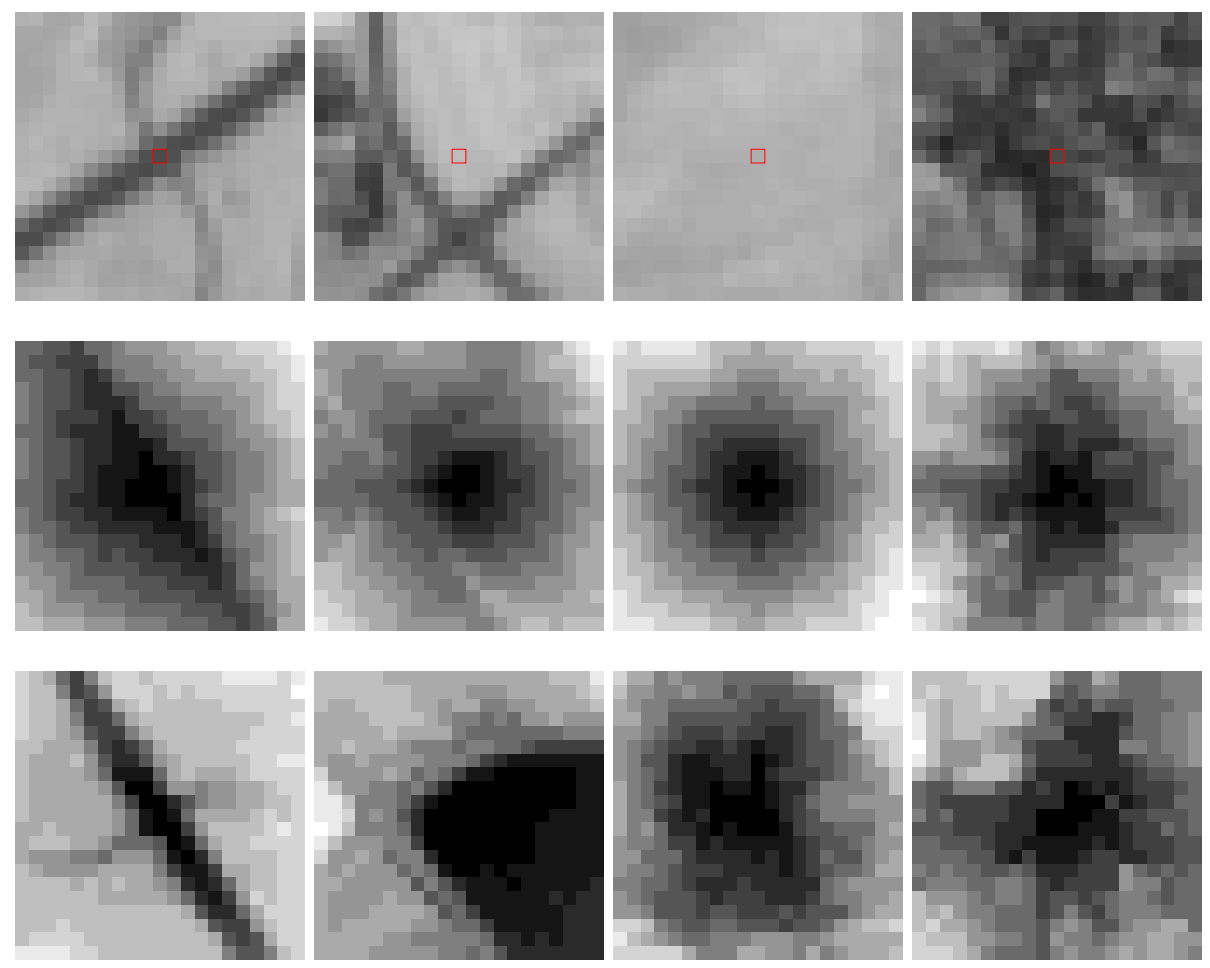

Fig. 1. Examples of adaptive geodesic neighbourhoods. Top: excerpt of a satellite image $(21 \times 21$ pixels $)$ with a marker pixel located in its center (red square); middle: geodesic time computed on the magnitude of the image gradient; bottom: ibid. on the image variations. From left to right, the marker pixel is resp. situated on a thin linear structure, near to a strong discontinuity, on a homogeneous textured area and in a noisy region. The estimated time was discretised in the range $[1,12]$ in order to visualise the different geodesic levels.

take advantage of the fact that images are finite and their intensity values in the monospectral case - are totally ordered. Therefore, they guarantee that pixels that effectively contribute to the output are processed only once.

\subsection{Adaptive Neighbourhoods}

The local estimation of discrete geodesic time at every pixel location enables to combine both spatial and tonal information into the definition of new adaptive neighbourhoods. Given an input image $f$, we are able to derive local adaptive neighbourhoods in $f$ using the previous approach. Namely, by considering at every single pixel location $\mathbf{x} \in f$ the geodesic time $\tau_{g}(\mathbf{x}, \cdot)$ estimated from $\mathbf{x}(\mathbf{x}$ is then said to be a marker) over the magnitude of the spatial gradient $|\nabla f|$, new neighbourhoods accounting for local image variability can be built. The 
underlying idea is that the geodesic paths associated to this new measure instead of the standard geometric distance - define the intrinsic neighbourhood relationship between the sample points when the $2 \mathrm{D}$ image is projected onto the 3D spatial-tonal domain. Indeed, the geodesic time expressed with Eq. (1) and the geodesic mask $g=|\nabla f|$ is going through the lowest values of the spatial gradient.

Similarly, we can define a geodesic measure that accounts for both the distance between pixels and the roughness of the 'height map', i.e. a measure of the shortest path drawn on the projection of the 2D image onto the spatial-tonal domain. For this purpose, we redefine the $\operatorname{cost} c_{i}$ of crossing pixels as:

$$
c_{i}=\frac{1}{2}\left|g\left(\mathbf{x}_{i}\right)-g\left(\mathbf{x}_{i+1}\right)\right|+\left|\mathbf{x}_{i}-\mathbf{x}_{i+1}\right|
$$

with the distance $\left|\mathbf{x}_{i}-\mathbf{x}_{i+1}\right|$ as before and the geodesic mask set to $g=f$. This definition is equivalent to the weighted distance on curves space of [23]. Its intuitive interpretation is that it represents the minimal amount of ascents and descents to be travelled to reach a neighbouring pixel. Indeed, the geodesic time $\tau_{g}(\mathbf{x}, \cdot)$ estimated with Eq. (2) minimises the changes in greylevel values. Note that in practise the geodesic mask is in fact constantly updated through the propagation of the geodesic time [2327]. The neighbourhoods defined this way coincide with the kernels defined by the morphological amoebas of 14 .

We can observe the way neighbourhoods adapt to the image context on the geodesic levels in Fig. 1] In particular, on the second column, the pixels across the road are not included in the closest neighbourhood of the marker as the cost of crossing the road is high when considering either the spatial gradient or the image variations. Thus, by considering such neighourhoods in filtering applications, the contributions of pixels from the other side of the edge will be suppressed. In other words, if a pixel is located near an edge, then pixels on the same side of the edge will have much stronger influence in the filtering: greylevel values from across a sharp feature are given less influence because they are penalised by the geodesic time functions. This is a desirable property of a filtering approach.

\section{Adaptive Geodesic Filtering}

\subsection{Similarity Measures and Filtering Kernels}

The most common strategy encountered in adaptive filtering consists in building local kernel functions over image regions according to their contents 29]: around a pixel $\mathbf{x}$ to be updated, one defines a kernel $\mathcal{K}$ with proper weights depending on the actual image variability in the neighbourhood of $\mathbf{x}$; filtering is then performed through a weighted average of local samples with $\mathcal{K}$. A critical issue is then how to account for image variability for generating the weights of the kernel.

A possible approach consists in adapting the local effect of the filter by using both the location of the nearby samples and their intensity values within the 
neighbourhood defined by the kernel $\mathcal{K}$. Accordingly, we propose to use the similarity measure based on the local geodesic time values for defining the weighs of $\mathcal{K}$. Precisely, the local similarity is measured in the neighbourhood of the marker $\mathbf{x}$ as a (monotonically) decreasing function $\Psi$ of the geodesic time $\tau_{g}(\mathbf{x}, \cdot)$ over the geodesic mask $g: \mathcal{K}(\mathbf{x}, \cdot)=\Psi\left(\tau_{g}(\mathbf{x}, \cdot)\right)$. This way, the filtering is performed along the geodesic paths going through $\mathbf{x}$ as defined by the selected geodesic function (and depending on the geodesic mask). A standard Gaussian can be used for the function $\Psi$, but other functions are not excluded. By introducing such a tonal weight in the kernel function, the mixing of different intensity populations is prevented and the effective sampling procedure is adapted locally to the image features such as edges. Employing Eq. (1) with $g=|\nabla f|$, the time necessary to travel between two pixels separated by high gradient values is higher than the time necessary to travel between two pixels separated by low gradient values. Therefore, higher weights are assigned to the nearby sample pixels that involve low gradient values along the minimal geodesic paths from $\mathbf{x}$, as compared to samples that are either far away from $\mathbf{x}$ or separated by high gradient values. Using Eq. (2) with $g=f$, higher weights are assigned to the nearby sample pixels which are linked to $\mathbf{x}$ and with similar greylevel values. Note moreover the difference in this latter case with the amoeba filtering [14: not only the domain defined by the geodesic neighbourhoods is used in the filtering procedure, but also the geodesic time values themselves as they define the weights of the samples in the kernel, unlike the amoebas which consider rank statistics instead. Indeed, in the proposed filtering techniques, the adaptive concept results from both the spatial adjustment of the operational kernel and the adjustment of the weights upon the kernel.

In practice, due to memory and computational limitations, the support of $\mathcal{K}$ is limited to a fixed size, i.e. sample pixels that are further away (in the spatial domain) than a given distance $\omega$ to the central pixel are not considered. Running the filtering procedure that calculates the local geodesic time from each single pixel in the image finally results in a total complexity of $O\left(n \cdot \Omega^{2} \log \Omega^{2}\right)$ where $n$ is the number of pixels of the input image and $\Omega=2 \omega+1$.

\subsection{Application to Multispectral Image Smoothing}

As a special case of filtering, image smoothing is a common preprocessing stage used to improve the visual appearance in an image, and to simplify subsequent image processing stages such as feature extraction, image segmentation or motion estimation [2]. Traditionally, the problem of image smoothing is to reduce undesirable distortions - due to the presence of noise or the quality of the image acquisition process - while preserving important features such as homogeneous regions, discontinuities, edges and textures 3928.

We consider the geodesic filters introduced in section 3 for edge-preserving smoothing of multispectral images. When dealing with multivalued image, the previous approach needs however to be adapted. In particular, the calculation of the gradient magnitude used by the first proposed filter needs to be attentively considered in order to take into account the actual multispectral information. A 

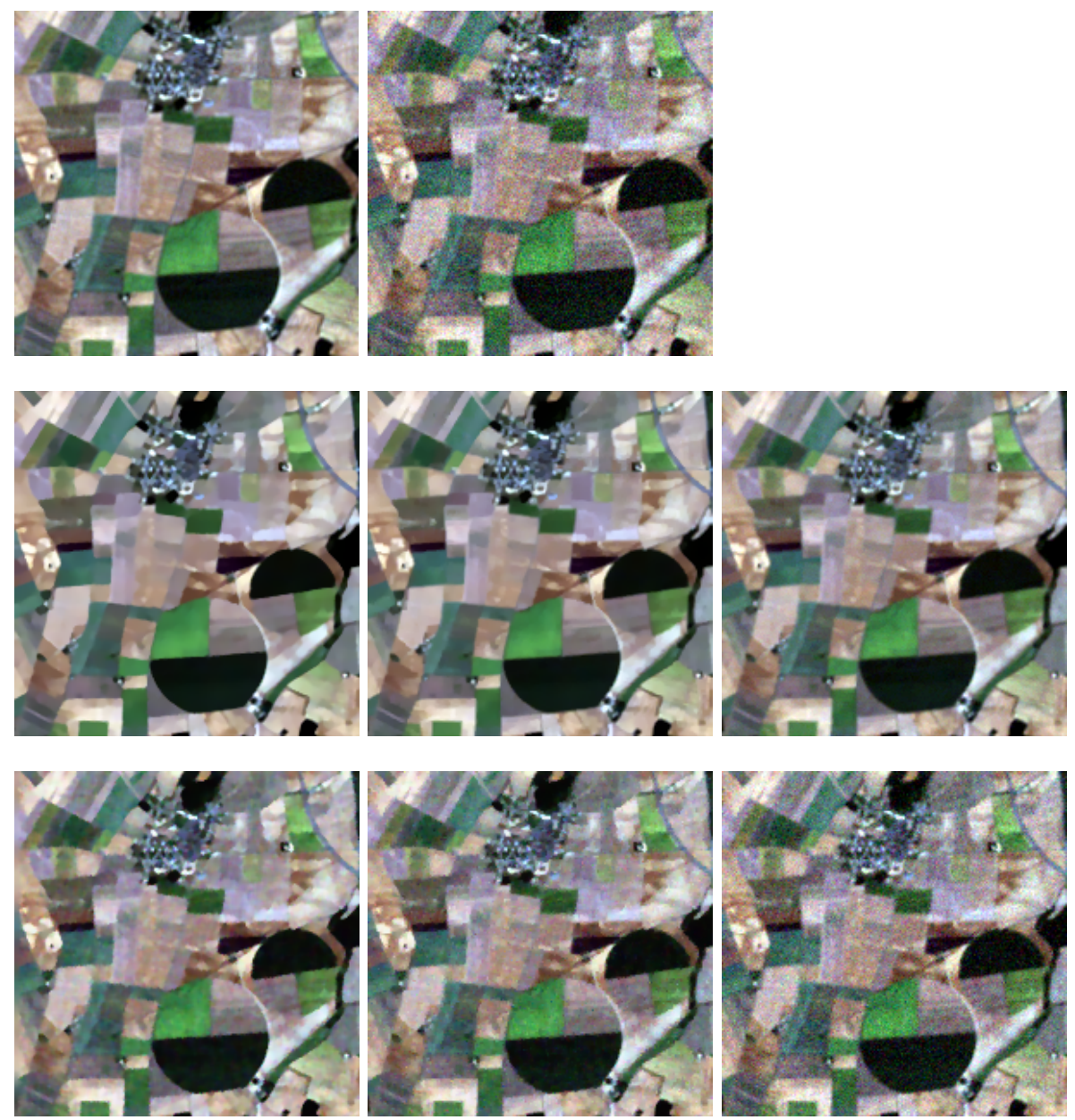

Fig. 2. Outputs of the proposed filtering approaches compared to the amoeba filtering of [14]. Results for a multispectral satellite image (top left) and its noisy version (Gaussian noise added, PSNR $=22.43 \mathrm{~dB}$ top right) are displayed on the second and third lines resp. From left to right: geodesic time computed on the magnitude of the image gradient, geodesic time computed on the image variations and morphological amoeba with median statistics. The parameters for the geodesic filters were set to $\alpha=10$ (second line) and $\alpha=7$ (third line); the window size was set to $\omega=7$. For comparison, the spatial extent of the amoeba kernels was similarly limited to a window of $7 \times 7$ pixels. PSNR values when filtering the noisy image: PSNR $=30.86 \mathrm{~dB}$ and $28.95 \mathrm{~dB}$ for the geodesic filters, and $27.57 \mathrm{~dB}$ for the amoeba filter.

way to estimate it for a multichannel image $f$ with components $f_{m}, m=1, \ldots, M$ is by means of the eigenvalue analysis of the image squared differential expressed by the $2 \times 2$ matrix: $\left[\sum_{m}\left(\frac{\partial f_{m}}{\partial x}\right)^{2}, \sum_{m} \frac{\partial f_{m}}{\partial x} \frac{\partial f_{m}}{\partial y} ; \sum_{m} \frac{\partial f_{m}}{\partial x} \frac{\partial f_{m}}{\partial y}, \sum_{m}\left(\frac{\partial f_{m}}{\partial y}\right)^{2}\right]$, the 
so-called first fundamental form [29]. The largest (postive) eigenvalue $\lambda$ is known to be the derivative energy in the most prominent direction (in particular, $\lambda=|\nabla f|^{2}$ for greylevel images with $M=1$ ) and is consequently a natural estimate for the gradient magnitude of the image. Therefore, we can apply Eq. (1) with $g(\mathbf{x})=\lambda(\mathbf{x})$. Approaches based on gradient information are however quite sensitive to noise. Indeed, noise corruption can generate discontinuities that are confused with other discontinuities that correspond to important features. Therefore, the local discontinuity measure based on the spatial gradient is not always robust. In Eq. (2), the norm must be understood as a multispectral norm, e.g the $L^{\infty}$ norm on the different channels; in such case, we have, when estimating the minimal path, $\left|g\left(\mathbf{x}_{i}\right)-g\left(\mathbf{x}_{i+1}\right)\right| \leq t$ if and only if $\left|f_{m}\left(\mathbf{x}_{i}\right)-f_{m}\left(\mathbf{x}_{i+1}\right)\right| \leq t$ for all $m=1, \ldots, M$; thus, the approach adopted by the second proposed filter depends on the dimension of the tonal space, which may increase its complexity.

Both filters were efficiently implemented for processing digital images using priority queues. They result in visually satisfying smoothed versions of the original images, blurring small discontinuities and sharpening edges (Fig. 2, bottom lines, first two columns). When compared with the morphological amoebas (Fig. 2, bottom lines, last column), they take advantage of the fact that the estimated geodesic time to the central pixel is also taken into account as a weighting function in the filtering procedure. Indeed, the generic filtering approach enables to conserve features through the combined spatial and tonal actions. Under that aspect, this approach can be linked to the bilateral filtering algorithm of [28]. Using a twofold similarity measure like the geodesic time, defined on either the spatial gradient or the image variation, enables moreover to account for the correlations between the positions of the pixels and their values, while bilateral filtering is breaking this correlation. Close inspection of the images also shows that the method is able to enhance texture regions. The degree of smoothing or sharpening can be further adjusted introducing a control parameter. Precisely, multiplying the $\operatorname{cost} c_{i}$ of crossing pixels by a given fixed value, say $\alpha$, results in amplifying or attenuating the local contrast in parts of an image. Small $\alpha$ values lead increasing the amount of blurring so that details are sacrificed, producing the well-known cartoon-like visual effect. With high values of $\alpha$, almost all contrasts are preserved and filtering has very little effect on the image. $\alpha$ will control the relative influence of tone and space in the calculation of the similarity measure of neighbour pixels. Other experiments have been conducted on benchmark natural images and compared with the outputs of some standard filtering techniques in 30 .

\section{Conclusion}

In this paper, we introduce new morphological adaptive operators for filtering. The adaptive concept results from both the spatial adjustment of the operators and the adjustment of the weights upon them. The basic idea is similar to that of spatial-tonal filtering approaches, which consist in employing both geometric and intensity closeness of neighbouring pixels. The originality of our 
approach lies in the definition of a new similarity measure combining both spatial and tonal information and based on the local estimation of some geodesic time functions. The proposed approach could be used as a preprocessing stage in feature extraction and/or image classification. Indeed, by blurring small discontinuities and sharpening edges, the image structures are not geometrically damaged, what might be fatal for further processing like classification or segmentation. Like other spatial-tonal based techniques, the degree of smoothing in the image can also be tuned. Further experiments should be led on strongly textured images and compared with other recently developed techniques.

Current research is geared towards improving and extending the present work. Improvements regard mainly the selection of the different parameters involved in the filtering strategy. A specific issue regards the spatial extent of the window used for estimating the local geodesic time functions (represented by $\omega$ herein). An alternative approach to the one adopted in this paper would be to limit the weighting average to the sample pixels reached from the central pixel with a time inferior to a given threshold value. This would simply be achieved by checking if the time is less than this threshold when we extract a new element from the priority queue and stopping the propagation process in such case. The role of the parameter $\alpha$ should also be studied more in depth.

The proposed approach is of particular interest for filtering data for which the discrete framework should be assumed, in order to avoid creating spurious artifacts through diffusion-like processes. We foresee further applications in the fields of remote sensing and medical imaging.

\section{References}

1. Fisher, R., Dawson-Howe, K., Fitzgibbon, A., Robertson, C., Trucco, E.: Dictionary of Computer Vision and Image Processing. Wiley, Chichester (2005)

2. Jähne, B.: Digital Image Processing: Concepts, Algorithms and Scientific Applications, 4th edn. Springer, Heidelberg (1997)

3. Saint-Marc, P., Chen, J., Medioni, G.: Adaptive smoothing: A general tool for early vision. IEEE Trans. Patt. Ana. Mach. Intel. 13, 514-529 (1991)

4. Paranjape, R., Rangayyan, R., Morrow, W.: Adaptive neighborhood mean and median image filtering. J. Elec. Im. 3, 360-367 (1994)

5. Pitas, I., Venetsanopoulos, A.: Nonlinear Digital Filters: Principles and Applications. Kluwer Academic Publishers, Norwell, USA (1990)

6. Nitzberg, M., Shiota, T.: Nonlinear image filtering with edge and corner enhancement. IEEE Trans. Patt. Ana. Mac. Intel. 14(8), 826-833 (1992)

7. Cheng, F., Venetsanopoulos, A.: Adaptive morphological operators, fast algorithms and their applications. Patt. Recog. 33, 917-933 (2000)

8. Debayle, J., Gavet, Y., Pinoli, J.C.: General adaptive neighborhood image restoration, enhancement and segmentation. In: Campilho, A., Kamel, M. (eds.) ICIAR 2006. LNCS, vol. 4141, pp. 29-40. Springer, Heidelberg (2006)

9. Mrázek, P., Weickert, J.J., Bruhn, A.: On robust estimation and smoothing with spatial and tonal kernels. In: Geometric Properties for Incomplete Data, pp. 335352. Springer, Heidelberg (2006)

10. Debayle, J., Pinoli, J.C.: General adaptive neighborhood image processing. J. Math. Im. Vis. 25(2), 245-284 (2006) 
11. Buades, A., Coll, B., Morel, J.M.: Neighborhood filters and PDE's. Num. Math. 105(1), 1-34 (2006)

12. Soille, P.: Morphological Image Analysis: Principles and Applications, 2nd edn. Springer, Heidelberg (2004)

13. Braga-Neto, U.: Alternating sequential filters by adaptive-neighborhood structuring functions. In: Mathematical Morphology and its Applications to Image and Signal Processing, pp. 139-146. Kluwer Academic Publishers, Dordrecht (1996)

14. Lerallut, R., Decencière, E., Meyer, F.: Image filtering using morphological amoebas. In: Proc. of ISMM. CIV, pp. 13-22. Springer, Heidelberg (2005)

15. Meyer, F., Maragos, P.: Nonlinear scale-space representation with morphological levelings. J. Vis. Comm. Im. Repres. 11(3), 245-265 (2000)

16. Soille, P.: Constrained connectivity for hierarchical image decomposition and partitioning. IEEE Trans. Patt. Ana. Mac. Intel (2008) (available online since October 2007)

17. Soille, P., Grazzini, J.: Advances in constrained connectivity. In: Proc. of DGCI. LNCS, vol. 4992, pp. 423-433. Springer, Heidelberg (2008)

18. Lavialle, O., Delord, D., Baylou, P.: Adaptive morphology applied to grey level object transformation. In: Proc. of ESPC, pp. 231-234 (2000)

19. Soille, P.: Generalized geodesy via geodesic time. Patt. Recog. Lett. 15(12), 12351240 (1994)

20. Lantuéjoul, C., Maisonneuve, F.: Geodesic methods in image analysis. Patt. Recog. 17, 177-187 (1984)

21. Verwer, B., Verbeek, P., Dekker, S.: An efficient uniform cost algorithm applied to distance transforms. IEEE Trans. Patt. Ana. Mach. Intel. 11(4), 425-429 (1989)

22. Coeurjolly, D., Miguet, D., Tougne, L.: 2D and 3D visibility in discrete geometry: an application to discrete geodesic paths. Patt. Recog. Lett. 25(5), 561-570 (2004)

23. Ikonen, L., Toivanen, P.: Distance and nearest neighbor transforms on gray-level surfaces. Patt. Recog. Lett. 28, 604-612 (2007)

24. Borgefors, G.: Distance transformations in digital images. Comp. Vis. Graph. Im. Proc. 34, 344-371 (1986)

25. Levi, G., Montanari, U.: A grey-weighted skeleton. Inform. Cont. 17, 62-91 (1970)

26. Cohen, L., Kimmel, R.: Global minimum for active contour models: a minimal path approach. Int. J. Comp. Vis. 24, 57-78 (1997)

27. Ikonen, L.: Pixel queue algorithm for geodesic distance transforms. In: Andrès, É., Damiand, G., Lienhardt, P. (eds.) DGCI 2005. LNCS, vol. 3429, pp. 228-239. Springer, Heidelberg (2005)

28. Tomasi, C., Manduchi, R.: Bilateral filtering for gray and color images. In: Proc. of ICCV, pp. 839-846 (1998)

29. Di Zenzo, S.: A note on the gradient of a multi-image. Comp. Vis. Graph Im. Proc. 33, 116-125 (1986)

30. Grazzini, J., Soille, P.: Edge-preserving smoothing of natural images based on geodesic time functions. In: Proc. of VISAPP, pp. 20-27 (2008) 